Acta arachnol., 43 (1): 49-55, June 30, 1994

\title{
Foraging in Cockroach Sticky Traps by the Spider Nesticodes rufipes LuCAS (Araneae: Theridiidae): A Super Food Resource
}

\author{
Bruce S. Cushing ${ }^{1)}$ and Lynn M. LeBeCK ${ }^{2)}$
}

\begin{abstract}
The spider Nesticodes rufipes regularly inhabits cockroach traps and feeds on entangled cockroaches. We determined, 1) the potential risks and gains associated with this foraging strategy and 2) whether spiders were evaluating the quality of traps before choosing which ones to inhabit. In terms of risk there was a $16 \%$ probability of a spider becoming entangled in a cockroach trap. The potential gains for spiders were in terms of food resources. Spiders that fed in cockroach traps were able to feed on significantly larger German cockroaches (Blattella germanica), than they could capture in naturally constructed webs, adult vs. 4th instars. Spiders also fed on adult American cockroaches (Periplaneta americana) in traps, which would represent an even larger potential gain in calories. When presented with a choice of traps containing one or five cockroaches, spiders displayed a significant preference for feeding in and inhabiting traps with more cockroaches. Traces of web were found in both traps indicating that the spiders were exploring their environment prior to choosing where to feed, and were actively selecting traps with more prey items. Cockroach traps represent a super food resource for $N$. rufipes, for several reasons: 1) There is a significant gain in available calories per prey item. 2) There are a large number of prey items available per unit area. 3) Prey items are available for an extended period of time, i.e. cockroaches in a trap remained alive on average almost 14 days after becoming entangled.
\end{abstract}

\section{Introduction}

Siders appear to use a wide variety of mechanisms for determining when and where to forage, including prey availability (Turnbull, 1964; GiLlesPie, 1981), the probability of capturing prey (OLIVE, 1982; VolLRATH, 1985), vegetation (RIECHERT and CADY, 1983), environmental conditions (EBERHARD, 1971; RIECHERT, 1976), and others (for review see RIECHERT and GILlesPIE, 1986). This suggests that, at least as a group, there is significant variability in foraging strategies, and that plasticity or rapid adaptation to novel situations may exist. The ability to respond to novel feeding situations could be particularly strong in spiders that are often found in association with human beings, because of our tendency to constantly manipulate our surroundings. However, foraging strategies, especially for web-building spiders are constrained by web construction and the size and type of prey they can effectively capture (Nentwig and Wissel, 1986; CraIG, 1987), regardless of the environment.

1) Department of Zoology, University of Hawaii, Honolulu, Hawaii 96822; current address: Biology Department, Middle Tennessee State University, Murfreesboro, Tennessee 37132, USA

2) Department of Entomology, University of Hawaii, Honolulu, Hawaii 96822, USA

Accepted June 2, 1994 
Given these factors, and the observation of Nesticodes rufipes LuCAS (Araneae, Theridiidae), a common spider in Hawaii and one that is frequently found in association with human dwellings (LEVI, 1967), feeding in cockroach sticky traps we attempted to determine: 1) Is this spider a frequent inhabitant of cockroach traps, i.e., regularly taking advantage of the potential food resource created by human activity? 2) Is there a potential gain for spiders that feed in these traps, that is, are they able to exceed the capabilities of their natural webs? 3) Is there any cost associated with this method of feeding? and 4) Are the spiders assessing their environment and selecting to feed in areas with a high density of available prey? Examining these questions in a spider feeding in a novel situation can provide further insight into foraging strategies and the decision process that foraging animals undergo.

\section{Methods}

A series of experiments were designed to determine: 1) How often are traps inhabited by spiders. 2) The potential costs and benefits associated with feeding in cockroach traps, and 3) If prey density is considered by the spiders in determining where to feed. All spiders used in the laboratory experiments were adult females that had been removed from ZORO ZORO cockroach traps. ZORO ZORO traps consist of a floor covered with a sticky substance and a central strip of attractive bait.

Trap survey. The purpose of the trap survey was to provide an estimate of when spiders begin feeding or residing in traps in relation to the number of food items available, and if the presence of cockroaches was necessary for a spider to inhabit a trap. Over a period of six months, 44 cockroach traps were placed in homes around the the island of Oahu and checked daily. We recorded the size and the species of trapped cockroaches, and the frequency of spider inhabitation and entrapment. No trap remained in use more than one month.

Spider capture efficiency. This experiment was designed to determine capture efficiency of $N$. rufipes and the maximum cockroach instar that could be handled in a natural web. Individual spiders were placed in $480 \mathrm{ml}$ plastic containers and allowed one week to construct a web. During this time spiders were provided a food source of either two adult leaf miners (Liriomyza sativae BLANCHARD, Diptera, Agromyzidae), or one first-instar German cockroach (Blattella germanica (L.), Blattaria, Blattellidae) every other day. On the eighth day a single German cockroach was placed in the bottom of the container. After $24 \mathrm{~h}$ we checked to determine if the cockroach had been captured, and if not, it was removed from the container. This procedure was repeated with 9 to 12 spiders for each cockroach instar from 1 st through 6th. The same spiders were used for each instar with a minimum of two weeks between trials. Percentage of individuals captured was recorded for each instar. Instar stage and mass of the cockroaches were determined prior to placement in the container.

Since N. rufipes had been observed feeding on American cockroaches (Periplaneta americana LinNAEUs, Blattaria, Blattidae) in cockroach traps, we weighed 10 adult American cockroaches to determine their average mass. We then used the average mass to generate an estimate of caloric content, providing a relative measure of potential energy available from feeding on these large cockroaches.

Prey-density choice tests. This experiment was designed to determine if prey- 


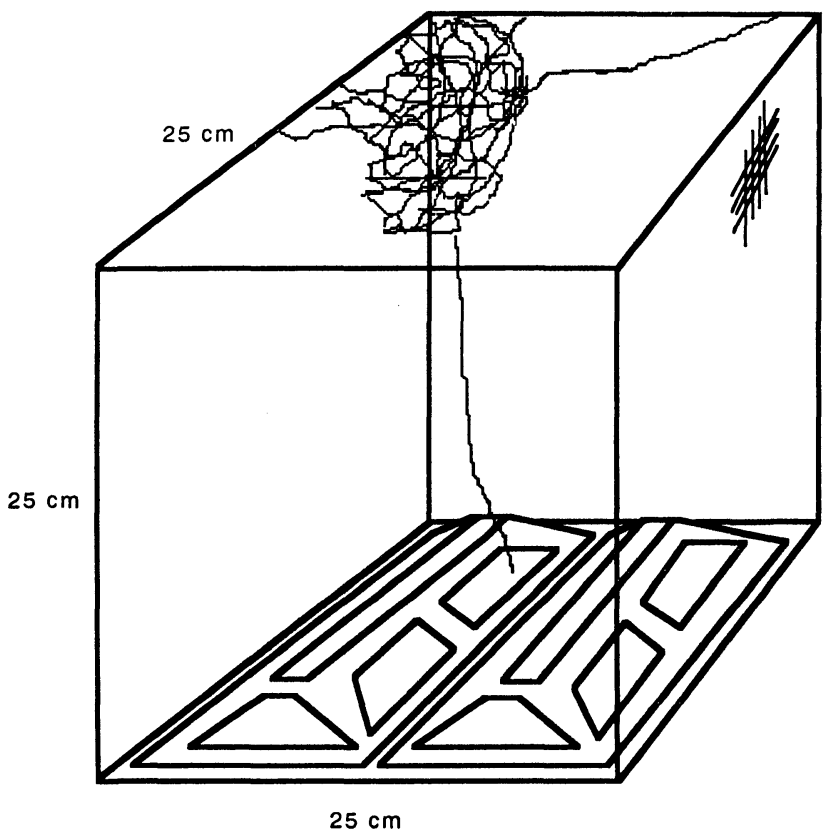

Fig. 1. Prey-density choice experiment, showing the cage and placement of cockroach traps. A web is protrayed in the upper corner of the cage, which was typical for most of the spiders.

density (number of prey available per trap) was a factor in trap selection. To test this we placed an individual $N$. rufipes in a cage measuring $25 \times 25 \times 25 \mathrm{~cm}$ (see Fig. 1). The front and the back of the cage were glass, with all other sides covered with a fine wire mesh, preventing the spider from leaving the cage. Spiders were allowed to acclimate to the cage for $24 \mathrm{~h}$, at which time two cockroach traps were placed in the bottom of the cage. One trap contained five adult German cockroaches while the other contained one adult. Placement of traps, one on the left side and one on the right side of the cage, was randomly determined. A test continued until a spider fed, at which time trap choice was recorded, or was itself trapped. Twenty-two spiders were tested, and for the purposes of analysis each trap was counted as a single unit.

Trapped prey longevity. To determine the potential long-term gain from inhabiting and feeding in cockroach traps, i.e. how long an individual prey item would be available, we quantified cockroach longevity in a trap. To accomplish this, ten adult German cockroaches were placed in two traps, five in each, and checked daily until they expired.

\section{Results}

Trap survey. Four species of cockroaches were captured in the traps during the survey: German (B. germanica (L.)), brown-banded (Supella longipalpa (F.)) 
(Blattaria, Blattellidae), American ( $P$. americana (L.)), and Australian cockroach ( $P$. australasiae) (F.)) (Blattaria, Blattidae). A total of 53 spiders were observed feeding in the 44 traps. During the period that traps were checked at least one spider was found in every trap with eight traps having more than one. One spider was found in a trap until a minimum of one cockroach had been trapped. In fact in a highly significant, 51 of 53, spiders fed from traps only after fiive or more lateinstar (5th or 6th) or adult cockroaches had been stuck. This strongly suggests that it is the presences of food items and not the traps themselves that are attracting the spiders. Seven of the fifty-three spiders were themselves caught in the cockroach traps $(13.2 \%)$.

Spiders in cockroach traps, appeared to feed predominantly on late instars or adults of all species of cockroach (over $95 \%$ of identifiable prey). Even though the cockroaches were entangled by the trap, spiders still webbed the cockroaches, and either fed on them stuck to the floor of the trap or pulled them off the surface to the trap ceiling or a nearby web. Eight female spiders produced from one to three egg sacs while inhabiting the traps. Shortly after hatching, spiderlings were observed feeding upon cockroaches. Although not censused, these and other spiderlings were observed feeding on all stages of cockroaches.

Capture efficiency. Within the container, using webs of their own construction webs, $N$. rufipes displayed a high capture rate of early instar German cockroaches. However, capture rate quickly dropped off, reaching zero by the 5 th instar (Fig. 2). This decline represents a significant difference in prey capture efficiency among instar stages $\left(\chi^{2}=35.28, d f=5, P<0.001\right)$. This indicates that in a natural web spiders are capable of capturing only relatively small prey items. Figure 2 also provides the estimated caloric content of the varying classes of German cockroaches. These values were obtained from WoODLAND et al.(1968) in which they determined caloric content using a bomb calorimeter.

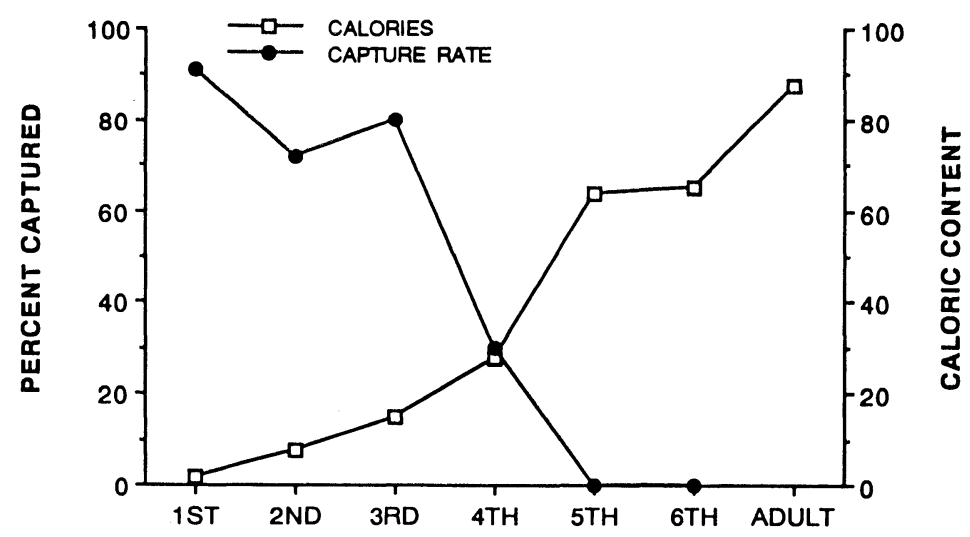

COCKROACH DEVELOPMENTAL STAGE

Fig. 2. Relationship between spider capture success rate and developmental stage of the German cockroach, and caloric content by instar (caloric content adapted from WoODLAND et al., 1968). 
Adult American cockroaches had a mean weight of $0.82 \mathrm{~g}(N=10$, S.E. \pm 0.135$)$.

Prey-density choice test. Within $4 \mathrm{~h}$ of being placed in a cage all spiders had initiated web construction in one of the upper corners. Nineteen of twenty-two spiders successfully fed. Sixteen of nineteen $(84.2 \%)$ selected a cockroach from the trap with five cockroaches (binomial distribution, $P<0.005$ ). Although most spiders established a web in the trap they fed in, three removed the prey item from the trap and pulled it towards their web in the upper corner. An examination of the traps revealed the presence of web in both traps, suggesting that the spiders were exploring their immediate environment before choosing to feed. Five of the twenty-two spiders were stuck in the traps, this includes three that did not successfully feed and two that were stuck after there initial feeding. This proportion did not differ from spiders stuck in traps placed in homes, 7 of $53\left(\chi^{2}=1.048, d f=1, n s\right)$. Because there was not a significant difference these results were combined, providing an estimated probability of $16 \%$ of a spider being stuck in a trap.

Trapped prey longevity. Adult B. germanica, in the two traps, survived an average of 13.65 (S.E. \pm 5.9 ) days. In both traps the maximum survival time was 23 days.

\section{Discussion}

The results of the trap survey, with at least one spider feeding in $100 \%$ of the traps, indicates that $N$. rufipes regularly uses insects trapped in cockroach traps as a source of food, and that while spiders may move through traps without prey that they only take up residency when prey is available. There are several benefits that $N$. rufipes appears to derive by feeding in cockroach traps. First, spiders are able to "capture" and feed on prey sizes and species that they cannot capture in natural webs. NenTwig and Wissel (1986) found that in many web-building spiders, prey size was limited to less than twice the length of the spider predator, with an upper limit of five times. If this holds true for $N$. rufipes then feeding in a cockroach trap results in a significant increase in the upper limit of prey size. In naturally constructed webs, the upper limit was 4th instar German cockroaches, with a capture efficiency of $30 \%$. The increase in upper size limit can be expressed in terms of potential caloric gain. If it is assumed that the portion of assimilable calories is the same for all stages of $B$. germanica, then feeding on entangled adults $v s$. 4th instars would represent on average more than a nine-fold gain in potential calories. The average potential gain is derived from the increase in available calories, 3 fold, times the increase in probability of capture. Furthermore, spiders were observed feeding on $P$. americana adults, which are much larger than adult $B$. germanica, and therefore feeding adult American cockroaches should represent even a large increase in potential available calories.

Second, the potential gain in food is magnified for spiders that take up residence in a trap, because prey items are available for an extended period of time. Although spiders appear to attack only living cockroaches, entangled adult German cockroaches remain viable for an average of two weeks, and as long as three weeks. This means that once a spider moves into a trap, trapped cockroaches will continue to be available, reducing a spider's need to relocate.

Cockroach traps may also represent a good place to raise offspring. Although 
a trap has a limited effective time, after which the floor loses its ability to trap animals, trapped cockroaches remain stuck. If a female spider were to produce an egg mass while in a cockroach trap, the spiderlings would develop as the trap aged and hatch after the trap had lost its adhesive qualities. However, trapped cockroaches would still be available for two weeks or longer, and spiderlings could feed upon the cockroaches without risk of being trapped. While not conclusive evidence, this scenario occurred four times.

Spiders use a variety of mechanisms for choosing a habitat, including prey availability (TurNBull, 1964; GILlesPie, 1981), vegetation (RIECHERT and CADY, 1983), environmental conditions (EBERHARD, 1971; RIECHERT, 1976), and others (for review see RIECHERT and GiLlesPIE, 1986). Nesticodes rufipes appears to assessing cockroach traps and making feeding choices based upon the availability of pray. First, no spiders were ever observed in residence in an empty cockroach trap, and rarely in one with less than five prey. Second, spiders were known to remove prey from the cockroach traps even when their webs were located outside of the traps, indicating that they had to have investigated the trap and chosen to feed on an entangled cockroach even though they were not residing in the trap at the time.

The remaining issue is whether or not there are risks associated with feeding in cockroach traps. Although there was a $16 \%$ chance of spiders becoming stuck in a trap, there are also unassessable "risks" such as when a trap is disposed of in the home environment. However, to determine how these risks compare with feeding in other environments it would be necessary to discover the risk of predation for $N$. rufipes not in cockroach traps. In conclusion, cockroach traps represent a super food resource for $N$. rufipes, and this spider appears to actively selected cockroach traps based upon the number of prey items.

\section{Acknowledgments}

We thank M. JoHnSON for his comments and help with certain aspects of this study. We also thank R. S. Gillespie, J. M. Cawthorn, C. R. Chandler, and N. Cushing for their critical comments on this manuscript.

\section{References}

Craig, C. L., 1987. The significance of spider size to the diversification of spider-web architectures and spider reproductive modes. Am. Nat., 129: 47-68.

Eberhard, W. G., 1971. The ecology of the web of Uloborus diversus (Araneae: Uloboridae). Oecologia, 6: 328-342.

Gillespie, R. G., 1981. The quest for prey by the web-building spider Amaurobius similis (Blackwall). Anim. Behav., 29: 953-954.

Levi, H. W., 1967. Habitat observations, record and new American theridiid spiders (Araneae, Theridiidae). Bull. Mus. comp. Zool., 136: 27-31.

Nentwig, W., \& C. Wissel, 1986. A comparison of prey length among spiders. Oecologia, 68: 595-600.

Olive, C., 1982. Behavioral responses of sit-and-wait predator to spatial variation in foraging gain. Ecology, 61: 1133-1144.

Riechert, S. E., 1976. Web site selection in the desert spider Agelenopsis aperta. Okios, 27: 311-315. 
Riechert, S. E., \& A. B. CADY, 1983. Patterns of resource use and tests for competitive release in a spider community. Ecology, 64: 899-913.

\& R. G. GILlespie, 1986. Habitat choice and utilization in web-building spiders. In SHEAR, W. A. (ed.): Spiders: webs, behavior, and evolution, pp. 23-48. Stanford University Press, Stanford, California.

Turnbull, A. L., 1964. The search for prey by a web-building spider Achaearanea tepidariorum (C. L. KoCH) (Araneae, Theridiidae). Can. Ent., 96: 568-579.

Vollrath, F., 1985. Web spider's dilemma: a risky move or site dependent growth. Oecologia, 68: $69-72$.

Woodland, D. J., B. K. Hall \& J. Calder, 1968. Gross bioenergetics of Blattella germanica. Physiol. Zool., 41 : 424-431. 